Spine:

Lumbar, thoracic, cervical facet joints,

intervertebral discs, interlaminar space Integumentary:

Dermis, hypodermis

Ocular:

Periocular soft tissues, conjunctiva, sclera,

episclera, cornea, uvea, lens, orbit, retina, optic

disc, vitreous body

Renal:

Renal parenchyma

(nephrolithiasis was excluded)

Cardiovascular:

Valves (mitral, pulmonic, aortic), myocardium,

conduction pathway, arterial wall, atherosclerotic

plaque

Gastrointestinal:

Small intestine, colon

Larynx

Breast

Middle Ear

Pancreas

Nasal

Prostate Gland

Liver

Pulmonary:

Bronchus

Penis

Nailbed

Pelvis maging
Autopsy, biopsy, surgery, imaging

Autopsy, biopsy, imaging

Clinical exam, biopsy

Biopsy, imaging

Clinical exam, biopsy, imaging

Biopsy, imaging

Clinical exam, biopsy Biopsy

Biopsy, imaging

Bronchoscopy, biopsy, imaging

Clinical exam, surgery

Clinical exam, biopsy Biopsy, imaging
Disclosure of Interests: Ada Kumar Shareholder of: I am an employee of Horizon Pharma and own shares in the company., Consultant for: I have worked as a paid consultant for Horizon Pharma., Employee of: I am currently employed by Horizon Pharma., Puja Khanna Grant/research support from: AstraZeneca, SOBI, Ironwood, Horizon, Consultant for: SOBI DOI: 10.1136/annrheumdis-2019-eular.1728

\section{AB0876 GOUT CAUSING URATE CARDIAC VEGETATIONS: SUMMARY OF PUBLISHED CASES}

Brian LaMoreaux ${ }^{1}$, Vidhya Chandrasekaran ${ }^{2} .{ }^{1}$ Horizon Pharma, Medical Affairs, Lake Forest, United States of America; ${ }^{2}$ Independent, Deerfield, United States of America

Background: Gout is a progressive inflammatory disease that is both widely prevalent and widely undertreated. In gout patients, urate deposition occurs in peripheral joints, the spine, and organs including the prostate, kidneys, and in atherosclerotic plaques as well as heart valves. Due to the concern of bacterial endocarditis and associated sequelae, cardiac valve vegetations are generally thoroughly evaluated even without systemic symptoms. Gout causing cardiac valvular vegetations is believed to be rare, though to date a number of case reports on this topic have been published.

Objectives: This project sought to compile and synthesize the existing published cases of proven cardiac valve urate deposition in gout patients. Methods: Medline and google were used to search for all available published cases involving gout associated with or causing cardiac valve vegetations. The references of each publication were additionally completely examined to find any other cases that may not have been identified on previous searches. The case reports were obtained and patient, disease, and valve factors were compiled and synthesized.

Results: Eight publications were found from 1954 to 2012 reporting 9 cases of urate deposition on cardiac valves. All cases had known tophaceous gout, mean age 60.9 , and $89 \%$ were male. The mean uric acid level was $10.2 \mathrm{mg} / \mathrm{dL}, 4 / 9$ (44\%) reported a heart murmur, and in only one $(11 \%)$ of the nine cases was infective endocarditis suspected. The diagnosis of gouty valvular involvement came from autopsy in 4 patients (44\%), pathological report in 3 patients (33\%), and was a diagnosis of exclusion after transthoracic echocardiogram in two patients $(22 \%)$. The mitral valve was the most commonly involved heart valve, with 6 of the 9 gout patients (66\%) with urate valvular deposits having them on the mitral valve. One patient had aortic and another had pulmonic valve involvement, while no patients were found to have tricuspid urate deposits from gout.

Conclusion: Though gout generally causes urate deposition in peripheral joints, urate deposits have been found in organs including the prostate, heart, kidneys, and eyes. Urate deposits from gout in heart valves are quite rarely reported, and this project describes 9 cases of urate valvular deposition associated with gout from 8 case report publications dating back to 1954. Based on these findings, urate deposition from gout on a cardiac valve will primarily remain a rare diagnosis of exclusion, but should be considered in patients with known longstanding visibly tophaceous gout, hyperuricemia, mitral valve involvement, and a lack of systemic signs or symptoms of infective endocarditis.

\section{REFERENCES}

[1] Traut, EF, et al, "Specific vascular changes in gout", JAMA, 1954,156 (6):591-593

[2] Dennstedt, FE, et al, "Tophaceous mitral valve: Report of a case", Am J of Surg Path, 1982;6(1):79-81

[3] Curtiss, El, et al, "Pulmonic regurgitation due to valvular tophi", Circulation, 1983:67(3):699-701

[4] Jaworski. RC, et al, "Tophaceous aortic valve: a case report", Pathology, 1983(15):197-9

[5] Scalpino, JN, et al, "Case report: mitral stenosis associated with valvular tophi", Mayo Clin Proc, 1984;59:509-512

[6] Gawoski, JM, et al, "Aortic valvular tophus: identification by X-ray diffraction of urate and calcium phosphates", J Clin Pathol, 1985;38:873-876

[7] lacobellis, G, et al, "A rare and asymptomatic case of mitral valve tophus associated with severe gouty tophaceous arthritis", J Endocrinol Invest 2004;27:965-966

[8] Rohani, A, et al, "A case of mitral valve tophus in a patient with severe gout tophaceous arthritis", J Clin Imaging Sci, 2012;2:68

Disclosure of Interests: Brian LaMoreaux Shareholder of: Horizon Pharma Employee of: Horizon Pharma, Vidhya Chandrasekaran: None declared DOI: 10.1136/annrheumdis-2019-eular.115

\section{AB0877 OXIDIZED LOW DENSITY LIPOPROTEIN IS CORRELATED WITH INFLAMMATORY STATUS IN GOUTY ARTHRITIS}

Kyung Ann Lee ${ }^{1}$, Suyeon Park ${ }^{2}$, Bo Young Kim ${ }^{3}$, Yun Sung Kim ${ }^{4}$, Jung Ran Choi ${ }^{5}$ Hyun-Sook Kim ${ }^{1}$. ${ }^{1}$ The Soonchunhyang University Seoul Hospital, Internal Medicine, Seoul, Korea, Rep. of (South Korea); ${ }^{2}$ The Soonchunhyang University Seoul Hospital, Biostatistics, Seoul, Korea, Rep. of (South Korea); ${ }^{3}$ Gangneung Asan Hospital, Internal Medicine, Gangneung, Korea, Rep. of (South Korea); ${ }^{4}$ Chosun University Hospital, Internal Medicine, Gwangju, Korea, Rep. of (South Korea); ${ }^{5}$ Pohang St. Mary's Hospital, Internal Medicine, Pohang, Korea, Rep. of (South Korea)

Background: Lipid peroxidation and oxidized LDL (oxLDL) are hallmarks in the development of various metabolic, cardiovascular and other inflammatory diseases. Cholesterol crystals and oxLDL, which are considerably present in atherosclerotic plaques, can activate inflammasomes. The concentration of plasma ox-LDL was also closely associated with the high incidence of atherosclerotic diseases in patients with gout might be closely related to increased ox-LDL levels. However, the study about the relationship between the level of ox-LDL and the inflammatory status in gout patients are rare.

Objectives: We aimed to the relationships between ox-LDL level, inflammatory markers and uric acid lowering agents in gout patients.

Methods: One hundred seventy four gout patients were included from the 3 institutions from 2014 to 2017. Details of demographic and clinical features along with laboratory parameters, ESR, CRP, lipid profiles, oxLDL and disease status were noted in patients with gout. We classified inflammatory status as acute, subacute chronic tophaceous and well controlled status. The level of ox-LDL was measured using the latex enhanced immune transmission turbidimetric method. We analyzed the comparison between dermographics, inflammatory markers, lipid profiles and disease subset.

Results: The primary objective of this study was to assess the oxLDL according to inflammatory status in gouty arthritis. Mean age was 50.4 year-old, $85.7 \%$ was woman, $98.9 \%$ were male, mean BMI was 25.86 and mean disease duration was 48 months. Among 174 gout patients 61 patients $(35.1 \%)$ were acute flare-up, 38 patients $(21.8 \%)$ were subacute, 67 patients (38.5\%) were chronic tophaceous, and 7 patients $(4 \%)$ were well controlled stable status.

Age, sex, BMI, and accompanying disease were not statistically different in the 4 groups according to inflammatory status. However, it showed statistically significant differences in the use of glucocorticoid and uric acid lowering agents. There was no significant difference in uric acid in laboratory tests, but there was a significant difference between ESR and CRP in each disease group. Especially, oxLDL showed difference according to inflammatory state of disease. 


\begin{tabular}{|c|c|c|c|c|c|}
\hline Laboratory features & Acute & Subacute & Chronic tophaceous & Is Well controlled & $p$-value \\
\hline Uric acid & $7.22 \pm 2.07$ & $6.77 \pm 2.40$ & $6.94 \pm 2.20$ & $7.24 \pm 1.65$ & 0.780 \\
\hline ESR & $36.36 \pm 32.52$ & $18.87 \pm 18.98$ & $16.85 \pm 18.30$ & $8.29 \pm 7.67$ & $0.000^{m+n}$ \\
\hline CRP & $1.45 \pm 2.31$ & $0.45 \pm 1.12$ & $1.33 \pm 4.58$ & $0.12 \pm 0.08$ & $0.008^{m+1}$ \\
\hline Lipid profiles & . & . & o & ? & . \\
\hline Total cholesterol & $193.9 \pm 46.06$ & $208.39 \pm 47.77$ & $185.21 \pm 49.08$ & $163.14 \pm 44.93$ & $0.042^{t}$ \\
\hline HDL & $46.10 \pm 11.96$ & $43.28 \pm 9.51$ & $43.58 \pm 12.98$ & $42.57 \pm 8.76$ & 0.539 \\
\hline Triglyceride & $216.85 \pm 171.69$ & $221.55 \pm 117.9$ & $207.05 \pm 108.78$ & $180.57 \pm 125.70$ & 0.645 \\
\hline LDL & $126.81 \pm 55.04$ & $126.05 \pm 41.27$ & $119.98 \pm 38.95$ & $111.4 \pm 25.68$ & 0.825 \\
\hline Oxidized LDL & $785.38 \pm 369.14$ & $775.5 \pm 272.80$ & $653.23 \pm 430.99$ & $366 \pm 173.43$ & $0.001^{+t+\infty}$ \\
\hline
\end{tabular}

Conclusion: The levels of oxLDL in gout patients were significantly different according to inflammatory status. The oxLDL may be associated with inflammation process in gout patients.

Disclosure of Interests: None declared

DOI: 10.1136/annrheumdis-2019-eular.7113

\section{AB0878 CARDIOVASCULAR GOUT}

Eleanora Mikhnevich ${ }^{1}$, Tatiana Pavlovich ${ }^{2} .{ }^{1}$ Belarusian State Medical University, Internal Medecine, Rheumatology, Minsk, Belarus; ${ }^{2}$ Belarusian State Medical University, Internal Medecine, Minsk, Belarus

Background: Gout is characterized by clinical heterogeneity and associated with multicomorbidity. In clinical practice, we can highlight a group of patients suffering from cardiovascular diseases (CVD) and requiring constant medication, in which the gout joins later.

Objectives: To study the group of patients with gout developing on the basis of pre-existing CVD at the gout onset.

Methods: 240 patients from our Center database with confirmed gout were included in our study. Comorbidities were registered before the gout onset, at its appearance. The study group consisted of 140 patients with CVD: hypertension with duration for 5 years and treated by medication, $\mathrm{CHD}$, atrial fibrillation, CHF and stroke. The comparison group consisted of patients with gout, but without CVD ( $n=100)$.

Results: Among patients with pre-existing CVD, the gout debuted later, at the age of $60(55-65)$ years ( $p 0.001)$. The most frequent comorbidity was $\mathrm{HTN}-83.6 \%(\mathrm{n}=117)(\mathrm{OR}=7.63 ; 95 \% \mathrm{Cl} 5.33-10.93 ; \chi 2=48.9, \mathrm{p}$ 0.001). Diabetes mellitus $(\mathrm{OR}=4.47 ; 95 \% \mathrm{Cl} 3.65-5.48 ; \mathrm{F}=0.035, \mathrm{p}=$ 0.005 ) also dominated in this group. At the same time, BMl $\geq 25 \mathrm{~kg} / \mathrm{m}^{2}$ was prevalent in comparison group $(\mathrm{OR}=19.46 ; 95 \% \mathrm{Cl} 2.84-133.2 ; \chi 2$ $=15.4$, p 0.001). Alcohol abuse was considerably lower $-37.1 \%(n=52)$ $(\mathrm{OR}=54.72 ; 95 \% \mathrm{Cl} 17.87-167.44 ; \chi 2=88.8, \mathrm{p} \quad 0.001)$, but medication use was higher - diuretics in $16.4 \% \quad(n=23) \quad(O R=6.36 ; 95 \% \mathrm{Cl} 5.28-7.64$; $\mathrm{F}=0.045, \mathrm{p} 0.001)$ and low-dose aspirin in $35.0 \% \quad(\mathrm{n}=49)$ of patients $(\mathrm{OR}=17.41 ; 95 \% \mathrm{Cl} 14.80-20.47 ; \mathrm{F}=0.147, \mathrm{p} 0.001)$. In the study group, the urolithiasis was evidenced more often $(\mathrm{OR}=4.13 ; 95 \% \mathrm{Cl} 3.42-4.98 ; \chi 2$ $=12.1, \mathrm{p}$ 0.001), and the presence of CKD with GFR $60 \mathrm{ml} / \mathrm{min} /$ $1.73 \mathrm{~m}^{2}$ was observed in $15.0 \%(\mathrm{n}=21)$ of patients $(F=0.069, p 0.001)$, but not found in the comparison group. The percentage of patients with metabolic syndrome (3 components according to ATP III) did not differ between the groups ( $p$ 0.05).

The number of patients having a concentration of uric acid (UA) in blood

$360 \mathrm{mkmol} / \mathrm{l}$ and $480 \mathrm{mkmol} / \mathrm{l}$ was similar in the both study and comparison groups ( $p$ 0.05). At the same time, the cases of UA 600 $\mathrm{mkmol} / \mathrm{l}$ were higher in study group by $13.1 \%(\mathrm{OR}=1.86 ; 95 \% \mathrm{Cl} 1.50-2$ .38; $\chi 2=5.94, p=0.015$ )

Conclusion: Among our patients, the gout developing on the basis of pre-existing CVD is one of the variant of a debuting gout. The group of patients with so called "cardiovascular gout" is characterized by later gout onset, comorbidities typical for CVD, prevalence of medication (low-dose aspirin, diuretic) and renal mechanisms-triggers of gout. In these patients, the maximal concentration of UA in blood ( $600 \mathrm{mkmol} / \mathrm{l}$ ) was registered more frequently than in patients with primary gout.

Disclosure of Interests: None declared

DOI: 10.1136/annrheumdis-2019-eular.4363

\section{$\mathrm{AB} 0879$ \\ INTRATENDINOUS TOPHI IN PATIENTS WITH GOUT: PERSISTENCE IN SPITE OF CLINICAL CONTROL AND SCOPE OF URICEMIA THERAPEUTIC TARGET}

Carlos Guillén-Astete, Patricia Morán-Alvarez. Hospital Ramón Y Cajal, MADRID, Spain

Background: The tendon affectation of the gout consists of the deposit of uric acid in territories habitually perientésicos causing its accumulation in the substance of the body of the tendon or occupying part of it and protruding towards zones of extension. Their presence is usually not clinically accounted for except when they obstruct tendon displacement and therefore their role as part of the clinical picture of patients with gout is often underrepresented.

Objectives: The purpose of this study is to determine the ultrasound response of these tophi once the clinical activity has been controlled and the serum uricemia therapeutic target of $6.0 \mathrm{mg} / \mathrm{dL}$ has been reached. Methods: Quasi-experimental study type before after. We include 19 patients with tophaceous gout diagnosed between 2012 and 2014 and followed regularly in three rheumatology consultations through clinical, analytical and ultrasound controls. We compared the ultrasound records of static images of your Achilles, patellar and tricipital tendons at the time of diagnosis and in your last review. The longitudinal measurements of the tophi obtained were compared. The independent variable was the evaluation time (before and after) and the dependent variables were the quantification of tophi and their measurements in the longitudinal axis.

Results: All the patients were male. Mean age of 58 SD 6.8 years (Range 29-76). Mean basal uric acid $11.3 \mathrm{mg} / \mathrm{dL}$. Mean follow-up time to reach therapeutic objective 18 SD 10 months (Range 9-61). Median number of intratendinous tophi (counting one for each tendon of 6 possible tendons): 2. Fashion 2 (Range 1-4). Mean longitudinal diameters: Achilles (8 cases) 18.3 SD 4.2 mm; Rotulian (11 cases) 15.3 SD $3.1 \mathrm{~mm}$; Tricipital (6 cases) $9.8 \mathrm{SD} 5.1 \mathrm{~mm}$. Hypouricemiant treatment administered: Alopurinol 12; Alopurinol followed by Febuxostat 7; Febuxostat only 0. In the ultrasound study, once the therapeutic objective was reached, the accounting of the intratendinous tophi Achilles and patellar tophi remained identical. One tricipital tofo disappeared completely and the rest were maintained. The longitudinal measurements of tophi that did not disappear were as follows: Achilles 18.0 SD $3.2 \mathrm{~mm}(\mathrm{P}=0.805)$; Rotulian $14.9 \mathrm{SD}$ $3.2(\mathrm{P}=0.803)$; Tricipital $7.9 \mathrm{SD} 3.2(\mathrm{~N}=5, \mathrm{P}=0.489)$. No differences were detected when comparing the two hypouricemiant treatment sequences.

Conclusion: Although this is a small retrospective cohort, our results show a poor ultrasound response of reduction of intratendinous tophi reaching the serum uricemia target of $6.0 \mathrm{mg} / \mathrm{dL}$. This observation raises two reflections: (1) On the one hand, and as already proposed, the therapeutic target of uricemia in our patients could require an even stricter adjustment and (2) on the other hand, it would be necessary to consider whether the inclusion of regulated ultrasound scans of certain tendons would have clinical value if they were routinely incorporated into the study of our patients with gout.

\section{DISCLOSURE OF INTERESTS}

None declared

DOI: 10.1136/annrheumdis-2019-eular.8206

\section{AB0880 ACUTE GOUTY ARTHRITIS RELATED EMERGENCY DEPARTMENT VISITS AMONG US VETERANS: CHARACTERISTICS, PREDICTORS AND AREAS OF IMPROVEMENT}

Debendra Pattanaik, Zunaira Ali, Amado Freire. University of Tennessee Health Science Center, Memphis, United States of America

Background: Gout is quite prevalent among United States veterans and many do not achieve optimal levels of uric acid $(<6 \mathrm{mg} / \mathrm{dl}){ }^{1}$ This suggests that there are large number of veterans who are at risk for gout flare, leading to utilization of health care resources such as emergency department (ED) and outpatient office visits. We investigated the ED visit patterns among veterans with history of gout and the factors contributing to ED visits.

Objectives: The objectives of the study were to identify the risk factors for ED visits by veterans for gout flare up. Future remediation of the risk factors would reduce utilization of health care resources.

Methods: This was a retrospective chart review of veterans diagnosed with gout in the ED at VA Medical Center Memphis TN between January $1^{\text {st }}, 2011$ and December $31^{\text {st }}, 2016$ using ICD-9 codes. A rheumatologist reviewed all cases and only confirmed cases of gout were included in the study. There were 2516 veterans seen for acute gout during the 